\title{
APPLICATION OF SMOOTHED PARTICLE HYDRODYNAMICS (SPH) IN NEARSHORE MIXING: A COMPARISON TO LABORATORY DATA
}

\author{
Soroush Abolfathi ${ }^{1}$ and Jonathan Pearson ${ }^{2}$
}

\begin{abstract}
A weakly compressible smoothed particle hydrodynamics (WCSPH) method is used to simulate the nearshore flow hydrodynamics. The wave induced dispersion and diffusion are determined for monochromatic waves with significant wave height of $0.12 \mathrm{~m}$ and the wave period of $1.2 \mathrm{sec}\left(\mathrm{S}_{\mathrm{op}}=5 \%\right)$ based on WCSPH wave dynamics. The hydrodynamics of WCSPH model are compared to the laboratory results obtained from series of LDA measurements. The overall mixing coefficients across the nearshore are determined from WCSPH hydrodynamics. The mixing coefficients obtained are compared with the values determined from a series of fluorometric studies performed in a large-scale facility in DHI, Denmark. The results show that the wave profiles are in good agreement with the experimental data. The WCSPH model is proven to be well capable of estimating the dispersion across the nearshore.
\end{abstract}

Keywords: WCSPH; Dispersion; Diffusion; nearshore mixing; LDA

\section{INTRODUCTION}

The nearshore zone experiences pollutant loading through both the shoreline and seaward boundaries. From the seaward boundary, pollutant loading is transported landward towards the surfzone by the so-called Stokes drift effect (Stokes, 1847). From the shoreline boundary, runoff pollution, which can contain faecal indicator bacteria and human viruses (Schiff et al., 2003) can drain into the surfzone. Consequently, pollution can congregate in the nearshore region, and as such, the water quality can affect the health of the general public who frequent beaches and thus is seen as a global problem (e.g. over a million beachgoers visit Santa Monica Bay beaches on a typical summer weekend - Schiff et al., 2003). However, the key mass exchange processes related to the pollutant transport and dilution within the nearshore water body are still not fully understood.

There have been comparatively few studies [Pearson et al., 2002 and 2009; Feddersen, 2006; Clark et al., 2010; Abolfathi \& Pearson, 2014] to investigate aspects of mixing under waves and currents in the nearshore zone. Few field studies have been undertaken using tracers. However, the lack of detailed hydrodynamic data has made it difficult to interpret the mixing processes. Bowen and Inman (1974) suggested that seaward of the breaker region, the turbulence generated by waves is small compared to the current-generated turbulence. Svendsen and Putrevu (1994) performed a numerical study and proved that the cross- shore current generated by wave activities dominated the transverse mixing. They suggested that cross-shore currents could exceed the contribution of the mixing caused by turbulent activity by an order of magnitude. However, there was only little data available to fully validate their findings. This brief review suggests that although advances have been made in understanding the mixing processes, still little hydrodynamic and fluorometric data is available.

The nearshore region is a dynamic and constantly evolving environment which is shaped by interacting processes encompassing different spatial and temporal scales. These processes are generated through complex interactions between wind, waves, currents and morphology. Predicting the mixing coefficient in the nearshore region is challenging due to quantifying the bed and surface generated sources of turbulence, the effects of Stoke's drift, the interaction of the periodic orbital motions of the waves and the shearing effect resulted from longshore currents (Svendsen \& Putrevu, 1994; Pearson et al., 2009; Abolfathi \& Pearson, 2014). There are good fundamental theories of mean velocities in the nearshore region (Longuet-Higgins \& Stewart, 1964). However, limited data are available from measurements. Mixing processes have been theoretically described in recent years, but there are relatively few experiments to validate those findings. Furthermore, due to the lack of data, coastal water quality models are limited in their predictive capability.

Spatially variable currents in the nearshore region have been investigated in a few studies by using a fixed frame of reference Eularian measurement technique. These measurements rely on deployment of an array of stationary sensors that measure the current properties from a fixed reference point. George et al. (1994) investigated turbulence in the surfzone by both laboratory and field studies as well as using fixed frame of reference at a specific location. Trowbridge \& Elgar (2001) utilized dual- sensor

\footnotetext{
${ }^{1}$ Flow Measurement \& Fluid Mechanics Research Centre, Coventry University, Priory Street, Coventry, CV1 5FB, UK

${ }^{2}$ School of Engineering, University of Warwick, Coventry, CV4 7AL, UK
} 
technique that removes the contamination by surface waves to study near-bottom surfzone hydrodynamics. They examined an approximate alongshore momentum balance between wind stress and near-bottom turbulent Reynolds stress. Trowbridge \& Elgar (2001) used an array of five upwardlooking Sontek Acoustic Doppler Velocimeters (ADVs) that was mounted on a low-profile frame, and measured three-dimensional velocity vectors in a sample volume with a spatial scale of $\sim 0.01 \mathrm{~m}$ (Elgar et al., 2005). Feddersen et al. (2007) studied cross-shore tracer's dispersion in the surfzone with Eularian approach over a range of time-scales and space-scales. Generally, Eularian techniques are sensitive to the number of sensors utilized to record the spatial scales of motion. This imposes restriction on the mixing and dispersion coefficients obtained from analysing the Eulerian measurements. However, also Lagrangian approaches such as dye tracers and drifter measurements have been employed in the study of mixing and dispersion in the nearshore.

Harris et al. (1963) was one of the first studies that investigate dispersion of dye by tracking fluorescent dye. Inman et al. (1971) and Bowen \& Inman (1974) investigated different aspects of the mixing due to wave and currents in the nearshore area by using dye tracers in the field experiments. They defined three different mixing mechanisms due to the surface waves including the processes associated with the wave motion seaward of the breaking point, the mixing due to the breaking wave and the large scale mixing due to the movement of water in longshore and rip currents.

Pearson et al. (2002) studied on-offshore mixing seaward of the breaker region using tracer measurements. Takewaka et al. (2003) studied longshore currents in Japan by investigating dye diffusion in the longshore current field. Grant et al. (2005) studied alongshore transport of pollution at Huntington Beach by dye experiments and faecal indicator bacteria monitoring. Grant et al. (2005) analysis showed that alongshore flux of surfzone water is at least 50 to 300 times larger than the crossshore flux of surfzone water. Clark et al. (2014) used fluorescent dye and aerial imaging technique to study the nearshore mixing.

Feddersen (2007) studied breaking wave induced cross-shore tracer dispersion in the surfzone. Spydell et al. (2007) carried out field experiments and utilized drifters to study about $200 \mathrm{~m}$ of the shoreline within the surfzone (at depths of less than $5 \mathrm{~m}$ ) and with the alongshore uniform bathymetry and waves. There is a large variation in the value of eddy diffusivity coefficient reported by the studies mentioned above (from $0.025 \mathrm{~m}^{2} \mathrm{~s}^{-2}$ [Takewaka et al., 2003] to $8 \mathrm{~m}^{2} \mathrm{~s}^{-2}$ [Inman et al., 1974]). Feddersen (2007) concluded that this large variation is due to the differences in the experimental methods and the difficulties of the dye tracing method. Pearson et al. (2009) carried out laboratory experiments using tracer and suggested that transverse mixing inside the surfzone is proportional to $H^{3 / 2}$.

Applications of Lagrangian drifters in studying the mixing and dispersion have been mainly limited to large-scale offshore and oceanic filed studies (Souza et al., 2014). Drifter technology has been adopted to measure diffusivity in estuaries and coastal waters (Tseng, 2001).

This study uses experimental and numerical techniques to elucidate the underlying mechanisms that lead to the mixing of neutrally buoyant pollutants in the coastal waters. The laboratory investigations were carried out in large-scale facility using LDA and fluorometric measurements. The numerical model was developed using a weakly compressible SPH approach.

\section{MIXING MECHANISMS}

The mixing coefficient in the on-offshore direction is defined in this study as the sum of advective shear dispersion $\left(D_{x}\right)$ and turbulent diffusion $\left(v_{t}\right)$ [Eq.1].

$$
E_{x}=v_{t}+D_{x}
$$

The turbulent diffusion could be determined from turbulent kinetic energy $(k)$ with the use of an appropriate turbulent length scale $(l)$. This study has determined the depth-averaged turbulent diffusion coefficient following the methodology of Svendsen (1987):

$$
v_{t}=l_{x} k^{\frac{1}{2}}
$$

The turbulent length scale is defined as a function of local water depth $(d)$ in the nearshore. For the broken waves in the nearshore the length-scale is 0.25-0.3d (Svendsen \& Putrevu, 1994). 
The advective shear dispersion mechanism in the nearshore consists of the non-uniform distribution of velocities in the water column in the on-offshore direction. A $N$-zone dispersion model based on mathematical solution of Chikwendu (1986) is developed to quantify the dispersion coefficients across the nearshore. The model considers two-dimensional flow and divides it into $N$ zones of well mixed parallel flow where each zone has a fractional thickness of $q$, average velocities $u_{j}$, concentration $c_{j}$ and longitudinal diffusivities $D_{x j}$. The advection diffusion equation can be written in coupled form for the $N$ zones of the parallel flow in the on-offshore direction (Eq.3):

$$
\begin{gathered}
\partial_{t} c_{1}=D_{x 1} \partial_{x}^{2} c_{1}-u_{1} \partial_{x} c_{1}+b_{12} \beta_{1}\left(c_{2}-c_{1}\right) \\
\partial_{t} c_{j}=D_{x j} \partial_{x}^{2} c_{j}-u_{j} \partial_{x} c_{j}+b_{(j-1) j} \beta_{j}\left(c_{j-1}-c_{j}\right)+b_{j(j+1)} \beta_{j}\left(c_{j+1}-c_{j}\right)
\end{gathered}
$$

for $j=2,3, \ldots, N-1$

$$
\partial_{t} c_{N}=D_{x N} \partial_{x}^{2} c_{N}-u_{N} \partial_{x} c_{N}+b_{(N-1) N} \beta_{N}\left(c_{N-1}-c_{N}\right)
$$

where, $\beta_{j}=\frac{h}{h_{j}}=\frac{1}{q_{j}}(j=1,2,3, \ldots, N)$ and $b_{j(j+1)}=\frac{2 D_{y j(j+1)}}{h^{2}\left(q_{j}+q_{j+1}\right)}(j=1,2, \ldots, N-1)$

$D_{y j(j+1)}$ is the average vertical diffusivity between two consequent zones ( $j$ and $\left.j+1\right)$. The above system of ODEs can be solved by use of Fourier transformation and large time exponent $\gamma(\lambda)$ and the shear dispersion coefficient for $N$ zones can be written as Eq. 4 :

$$
\begin{aligned}
& D(N)=\sum_{j=1}^{N-1}\left(q_{1}+q_{2}+\ldots+q_{j}\right)^{2}\left[1-\left(q_{1}+q_{2}+\ldots+q_{j}\right)\right]^{2} \times\left[u_{12 \ldots j}-u_{(j+1) \ldots N}\right]^{2} / b_{j(j+1)}+ \\
& \sum_{j=1}^{N} q_{j} D_{x j}
\end{aligned}
$$

where, $u_{12 \ldots j}$ is the mean velocity in the first $j$ zones and $u_{(j+1)} \ldots_{N}$ is the mean velocity in the last $N-j$ zones. If the number of these zones tends to infinity, the thickness of each zone becomes infinitesimal and therefore the summations turn into the integral function. When $N$ tends to infinity, the approximation of $N$ zones model becomes an exact mathematical solution. The vertical variation of diffusivity parameter is considered for determining the shear dispersion coefficient for both laboratory and numerical study by estimating eddy viscosity for over 20 vertical points at every on-offshore location across the nearshore. For the numerical model the temporal variations of advective shear dispersion is determined using the methodology developed by Abolfathi (2017).

The theoretical on-offshore mixing mechanisms described in this section are adopted to quantify the nearshore mixing from laboratory investigations and numerical modelling. The results of hydrodynamic study are compared to fluorometric tracer studies.

\section{LABORATORY INVESTIGATIONS}

The experimental study has been performed by means of both Eulerian and Lagrangian approaches in the shallow water basin at the DHI (Pearson et al., 2006). A detailed hydrodynamic measurement in the nearshore region is performed by using Laser Doppler Anemometry (LDA) method. Fluorometric dye tracing study is carried out by use of Rhodamine water tracer dye to quantify the mixing coefficient under combined effects of waves and currents. The measurement section was 18x18 $\mathrm{m}$ and all the tests were performed on a 1:20 plain beach slope with an offshore water depth of $0.7 \mathrm{~m}$. The bed of the facility consists of concrete screed with an assumed roughness element $1 \mathrm{~mm}$ high. The facility was equipped with an absorbing piston-type wave-maker. This study presents monochromatic long crested waves in the nearshore. Table 1 summarizes the experimental study presented in this paper. 
Table 1 - Summary of experimental study

\begin{tabular}{ll}
\hline \multicolumn{1}{c}{ Current + Regular waves Hnominal $=0.12 \mathrm{~m}, \mathrm{~T}=1.2 \mathrm{~s} \& \mathrm{~S}_{\mathrm{op}}=5 \%$} \\
\hline Vertical LDA profiles (y-z direction) $\{\mathrm{y}\}$ & $1.08,2.08,3,4,5 \& 6 \mathrm{~m}$ \\
Vertical LDA profiles (x-z direction) $\{\mathrm{y}\}$ & $3 \& 5 \mathrm{~m}$ \\
Transverse dye profiles $\{\mathrm{y}\}$ & $2,3,5 \& 5$ bed $\mathrm{m}$ \\
Vertical dye profiles $\{\mathrm{y}\}$ & $5 \mathrm{~m}$ \\
\hline
\end{tabular}

Hydrodynamic measurements were undertaken at six on-offshore locations from the shoreline with a $1 \mathrm{~m}$ interval. For each on-offshore location a minimum of 20 points in vertical direction is studied. Fig. 1 shows the temporal averaged cross-shore velocity profiles inside the surfzone and seaward of the breaker region.

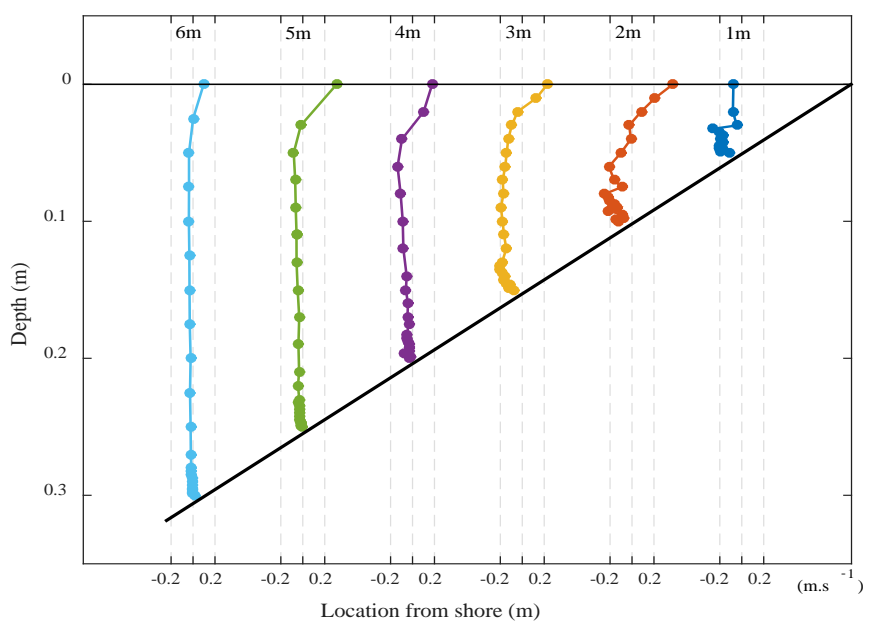

Figure 1 - Temporal averaged Cross-shore velocity for monochromatic waves with $S_{o p}=5 \%$

Fluorometric measurements were carried out using a constant injection of Rhodamine tracer dye at mid-depth. The transverse mixing characteristics have been determined at three distinct locations including the surf-zone, breaker-point, and offshore, which correspond to $y=\{2,3,5\} m$ from the shore line, respectively. Fig. 2 presents the tracer measurement results across the nearshore for the monochromatic waves of $5 \%$ steepness.

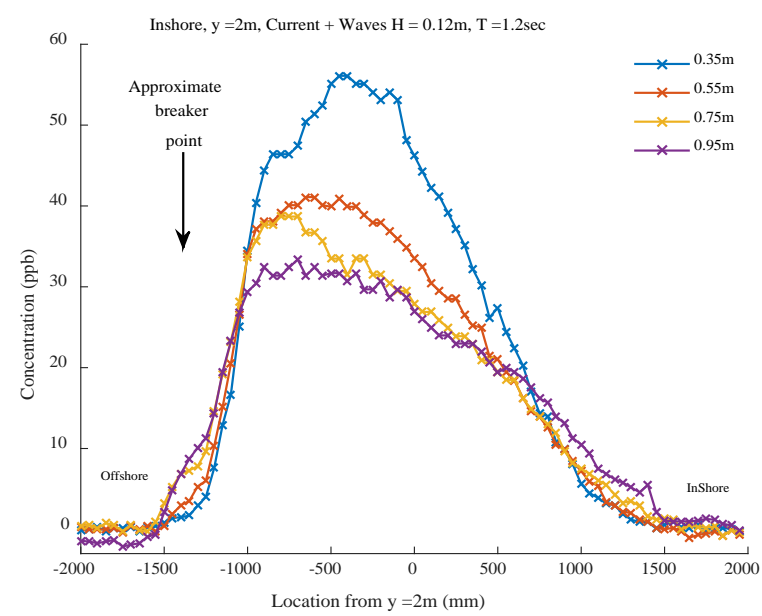



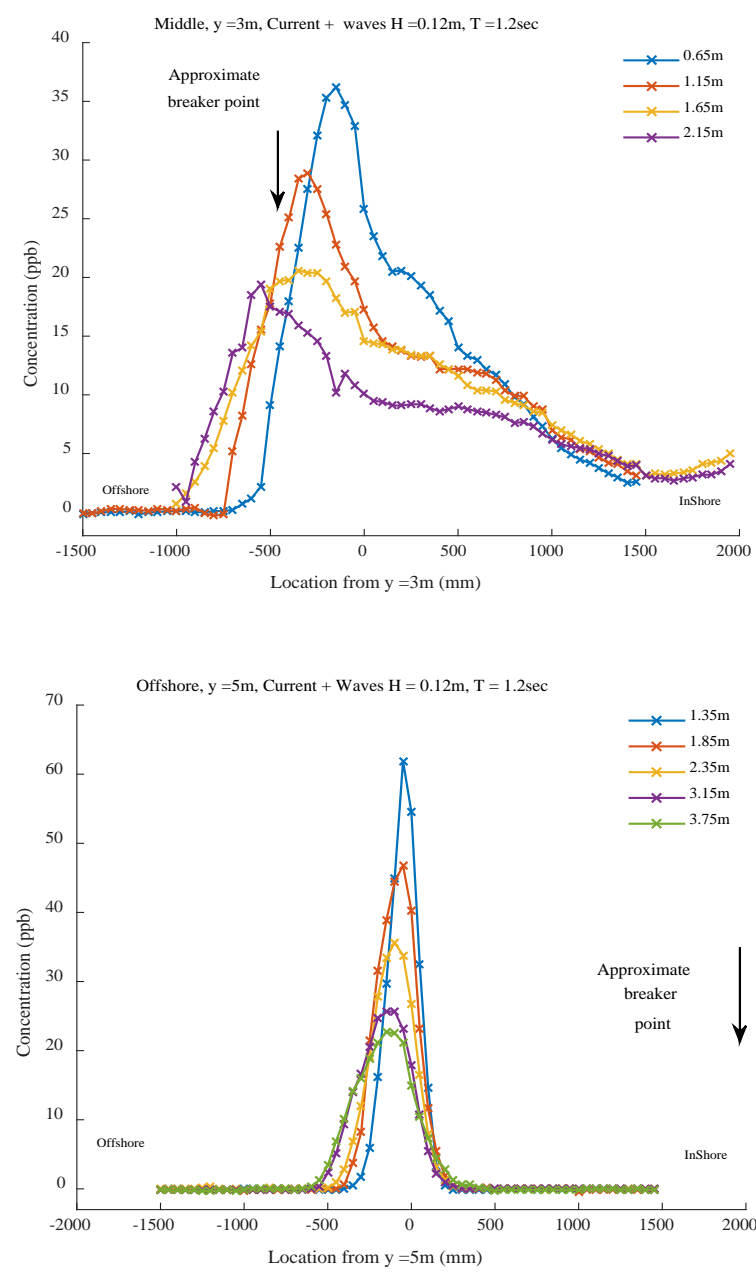

Figure 2 - Concentration for 3 injection points; surfzone, breaker point and offshore Wave condition monochromatic waves with $\mathrm{H}=0.12 \mathrm{~m}$ and $\mathrm{T}=1.2 \mathrm{sec}$

The variance of transverse concentrations against the distance from the injection points is determined (Fig. 3).

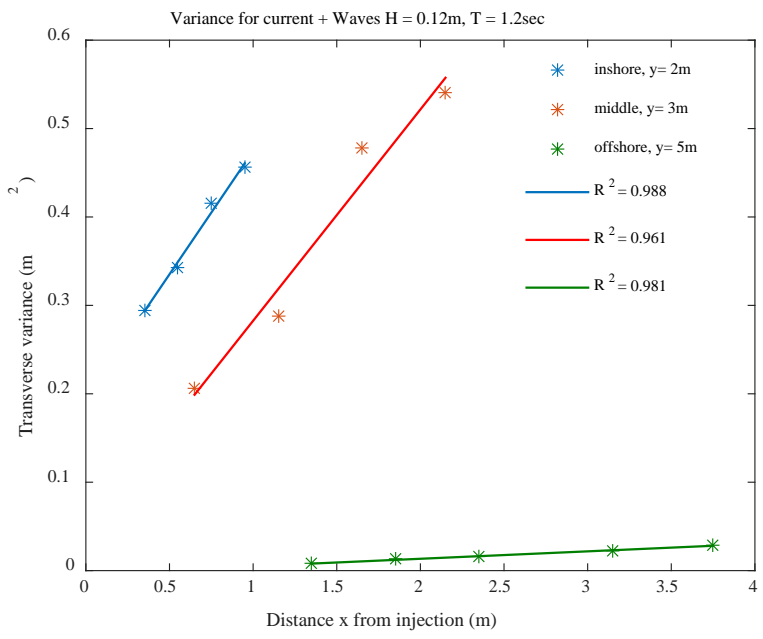

Figure 3 - Relationship between the variance of the transverse concentration profiles and longitudinal distance 
The tracer data is analyzed using Taylor (1954) methodology which combines the diffusive and dispersive mixing mechanisms. Table 2 summarized the analysis of tracer data at offshore, breaking region and inner-surfzone.

Table 2 - Transverse mixing results for a continuous injection of tracer at offshore, breaking region and inside the surfzone for monochromatic waves of $S_{o p}=5 \%$

\begin{tabular}{cccccccc}
\hline $\begin{array}{c}\text { Dye Injection } \\
\text { point } \\
{[\mathrm{m}]}\end{array}$ & $\begin{array}{c}\text { Offshore } \\
\text { wave height } \\
\mathrm{H}_{\mathrm{o}}[\mathrm{m}]\end{array}$ & $\begin{array}{c}\text { Offshore } \\
\text { wave Period } \\
\mathrm{Tp}[\mathrm{sec}]\end{array}$ & $\begin{array}{c}\text { Long. } \\
\text { velocity } \\
\left(\mathrm{U}_{\mathrm{d}}\right) \\
{[\mathrm{m} / \mathrm{s}]}\end{array}$ & $\begin{array}{c}\text { Bed shear } \\
\text { velocity } \\
{[\mathrm{m} / \mathrm{s}]}\end{array}$ & $\frac{d \sigma_{y}^{2}}{d x}$ & $\begin{array}{c}\text { Transverse } \\
\text { dispersion } \\
\text { coefficient } \\
\mathrm{D}_{\mathrm{y}} \\
{[\mathrm{m}]}\end{array}$ & $\frac{\left.\mathrm{m}_{y} / \mathrm{s}\right]}{d u^{*}}$ \\
\hline & 0.122 & 1.20 & 0.09 & 0.0045 & 0.279 & $1.195 \mathrm{E}-02$ & 27.9 \\
3 & 0.122 & 1.20 & 0.15 & 0.0075 & 0.239 & $1.261 \mathrm{E}-02$ & 15.9 \\
5 & 0.122 & 1.20 & 0.165 & 0.00825 & 0.0084 & $6.930 \mathrm{E}-04$ & 0.36 \\
\hline
\end{tabular}

\section{NUMERICAL MODEL}

In this paper, a two-dimensional Lagrangian particle-based model is developed based on the weakly compressible smoothed particle hydrodynamics (WCSPH) formulations. The governing equations for free surface flow simulation in two-dimensional Lagrangian formalism are:

$$
\begin{gathered}
\frac{1}{\rho} \frac{d \rho}{d t}+\nabla \cdot \vec{u}=\mathrm{C} \\
\frac{d \vec{u}}{d t}=-\frac{1}{\rho} \nabla P+\frac{1}{\rho} \nabla(\mu \cdot \nabla \vec{u})+\frac{1}{\rho} \nabla \cdot \tau+\bar{g}
\end{gathered}
$$

where $u$ is velocity vector, $P$ is pressure, $\rho$ represent density and $\mu$ is the kinematic viscosity. $\tau$ in Eq. 6 is the Sub-Particle Scale (SPS) stress tensor (Gotoh et al., 2001). The Navier-Stokes equations closured with SPS turbulence model are described in SPH notions in Eq. 7 \& 8.

$$
\left(\frac{d \rho}{d t}\right)_{a}=\sum_{b} m_{b} \vec{u}_{a b} \cdot \nabla W_{a b}
$$

$$
\begin{aligned}
& \left(\frac{d u}{a t}\right)_{a}=-\sum_{b} m_{b}\left(\frac{P_{a}}{\rho_{a}^{2}}+\frac{\rho_{b}}{\rho_{b}^{2}}\right) \nabla W_{a b}+\sum_{b} \frac{4 m_{b} \mu r_{a b} \cdot \nabla W_{a b}}{\left(\rho_{a}+\rho_{b}\right)\left(r_{a b}^{2}+\phi^{2}\right)} \vec{u}_{a b}+\sum_{b} m_{b}\left(\frac{\tau_{a}}{\rho_{a}^{2}}+\right. \\
& \left.\frac{\tau_{b}}{\rho_{b}^{2}}\right) \nabla W_{a b}+\vec{g}
\end{aligned}
$$

where $a$ and $b$ denote respectively the reference particle and its neighbors, $\vec{u}_{a b}=\vec{u}_{a}-\vec{u}_{t,}, \vec{r}_{a b}=\vec{r}_{a}-\vec{r}_{h}$, and $\varphi$ is a small number introduced to keep the denominator non-zero and usually is $0.1 h_{a b}$.

In order to overcome incompressibility problems associated with ISPH models, this study is solving the equation of state (Eq.9) instead of Poisson equation to approximate pressure field.

$$
P=B\left[\left(\frac{\rho}{\rho_{0}}\right)^{\gamma}-1\right]
$$

where $\gamma=7$ is a constant, $B=c_{0}^{2} \rho_{0} / \gamma$ in which the $\rho_{\mathrm{n}}$ is the reference density, $c_{\mathrm{n}}$ is the speed of sound at the reference density, and $c_{n}=c\left(\rho_{0}\right)=\sqrt{\partial P / \partial \rho}$.

Particles are moving within the numerical domain according to XSPH approach (Monaghan, 1994) [Eq. 10] to ensure neighboring particles will move with approximately the same velocity: 


$$
\left(\frac{d r}{d t}\right)_{a}=\vec{u}_{a}+\varepsilon \sum_{b} \frac{m_{b}}{\bar{\rho}_{a h}}\left(\vec{u}_{b}-\vec{u}_{a}\right) W_{a b}
$$

Where the constant $\varepsilon$ is 0.5 and $\bar{\rho}_{a b}=\left(\rho_{a}+\rho_{b}\right) / \check{L}$ and $W$ represents the weighting function or kernel. The last term in the equation is called XSPH correction, which guarantees that the neighboring particles possess approximately the same velocity components [Monaghan, 1994].

The numerical model is developed by using Yeganeh-Bakhtiary et al. (2017) methodology and adopting Cubic spline kernel function and Euler Predictor-Corrector time integration scheme. In order to overcome SPH particle deficiency near the boundaries, Dynamic boundary conditions (Crespo et al., 2007) is implemented for the numerical model. Madsen (1971) second order wave maker methodology was adopted for wave generation in order to prevent from spurious secondary waves. The numerical domain and the wave conditions are the same as the experimental conditions reported in this study.

The numerical model is used to simulate flow hydrodynamics across the nearshore for similar hydrodynamic conditions as the laboratory studies. The mixing and dispersion across the nearshore is determined from WCSPH model using a windowing technique to extract flow hydrodynamics at longitudinal locations and vertical points (Abolfathi, 2017).

\section{RESULTS \& DISCUSSIONS}

This section quantifies the mixing mechanisms described earlier by using the laboratory and numerical model hydrodynamic results.

\section{Diffusion Coefficient}

A full understanding of turbulent motion is one of the most challenging and intractable problems of the physical sciences. By adopting Reynolds analogy, the turbulent diffusion coefficient in the transverse direction $\left(e_{y}\right)$ is of similar magnitude to the eddy viscosity. Thus for the purposes of this present study it is assumed that the two variables are of the same magnitude. Hence, estimates of eddy viscosity values are usually derived from velocity measurement. Turbulence is dominated by breaking wave effects in the surfzone. Seaward of the breaker point turbulence can be generated from two sources; the first is from the residual turbulence generated from the breaking, where the turbulence generated in the surfzone, is transported seawards by the undertow. A second source of turbulence is caused by the oscillatory wave motion flowing over the bed.

The hydrodynamic data from laboratory measurements are utilized to determine the eddy viscosity according to Eq. 2. The turbulent kinetic energy $(k)$ is determined using two-dimensional velocity records from LDA measurements. The spurious data were detected and removed from the dataset by using a phase-space thresholding method (Goring \& Nikora, 2002). The TKE is determined by calculating the mean $(u)$ and turbulent velocity $\left(u^{\prime}\right)$ for each velocity wavelet. The individual wavelets are recognized using a zero-crossing algorithm. The mean velocity was determined by fitting Fourier series to LDA signal for each individual wavelet (Abolfathi, 2017). Fig. 4 shows the TKE results for LDA data for Monochromatic waves with $\mathrm{S}_{\mathrm{op}}=5 \%$.
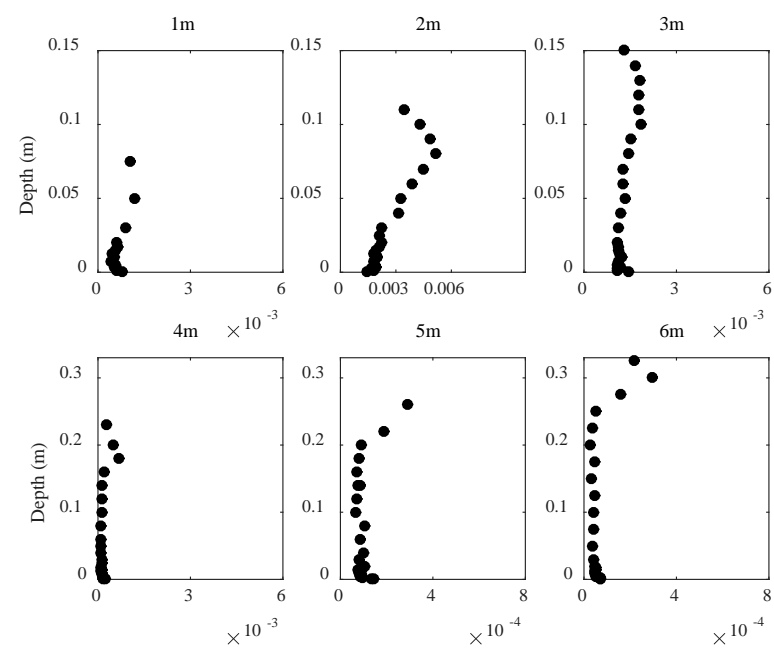

Turbulent Kinetic Energy $\left(\mathrm{m}^{2} . \mathrm{s}^{-2}\right)$

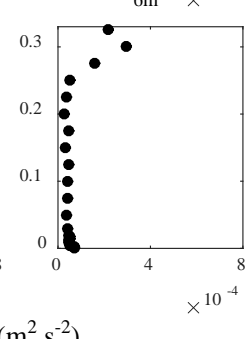

Figure 4 - Vertical structure of TKE across the nearshore from LDA data for the monochromatic waves of $S_{\text {op }}=5 \%$ 
The results for the turbulent kinetic energy estimated from LDA data are compared to the TKE measurements reported by Nadaokah \& Kondoh (1982) [Fig. 5]. Nadaokah \& kondoh (1982) undertook a series of hydrodynamic measurements in a laboratory flume with a plane 1:20 beach slope using the LDA technique and determined TKE using spectral analysis method.

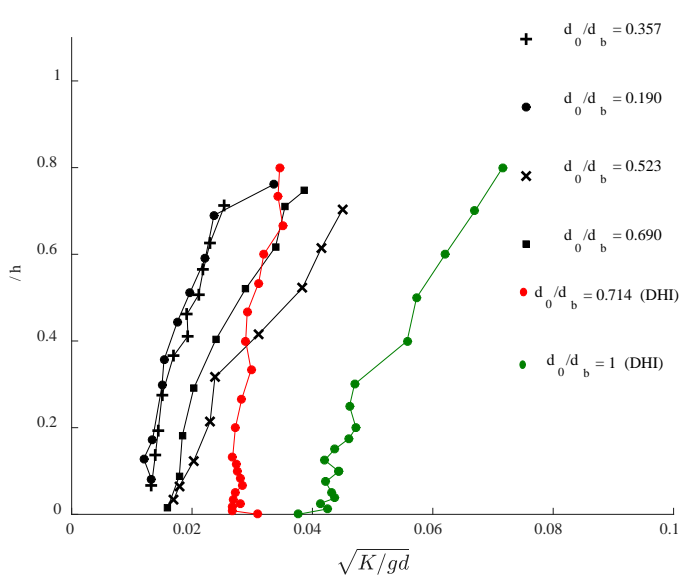

Figure 5 - Comparison of TKE reported by Nadaokah \& Kondoh (1982) with the laboratory measurements (DHI) for the case of monochromatic waves with $S_{o p}=5 \%$

The eddy viscosity is determined by using the depth averaged TKE and a constant length-scale of 0.3d. Fig. 6 presents the comparison between the eddy viscosities obtained from LDA measurements with the theoretical values proposed by Svendsen \& Putrevu (1994).

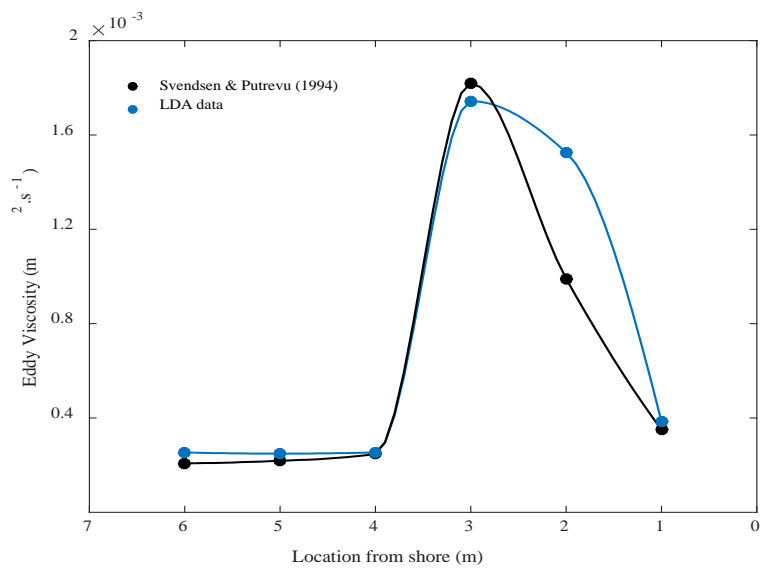

Figure 6 - Comparison of measured eddy viscosity from LDA data with Svednsen \& Putrevu (1994) theoretical formulae

\section{Dispersion Coefficient}

Wave kinematics and the hydrodynamics obtained from LDA measurements and WCSPH simulations are utilized to quantify the mixing coefficients across the nearshore using the $N$-zone model (Eq. 4).

The mixing coefficient was determined for five locations $(y=\{1,2,3,4,5\} m)$ across the nearshore with use of the hydrodynamics obtained from LDA and WCSPH model. Depth-averaged diffusivities calculated for LDA data and the theoretical values derived by Svendsen and Putrevu (1994) [Eq. 11] are adopted to determine advective shear dispersion coefficients for Laboratory data and numerical simulation, respectively.

$$
\begin{aligned}
& v_{t}=M d \sqrt{g d} \\
& v_{t}=\left(0.8\left(d / d_{b}\right)^{-4}+0.2\right) v_{t b}
\end{aligned}
$$


Eq. 11.a determines the diffusivity inside the surfzone and Eq. 11.b is valid seaward of the breaker region. A constant value of $M=0.03$ in Eq. 11.a was adopted for the numerical analysis. For each onoffshore location across the nearshore, the temporal variations of flow hydrodynamics from the WCSPH model were utilized with a depth-averaged eddy viscosity value to run the $N$-zone model. To extract the WCSPH velocity profile at each study location, a numerical window was defined in the model. Velocity profiles for WCSPH were calculated by spatially-averaging particle velocities at small measurement boxes $50 \times 50 \mathrm{~mm}$ from the bed to the top of the numerical domain. The velocity profiles were calculated for each numerical time-step. Fig.7 is the schematic of velocity profile calculations from the WCSPH simulations.

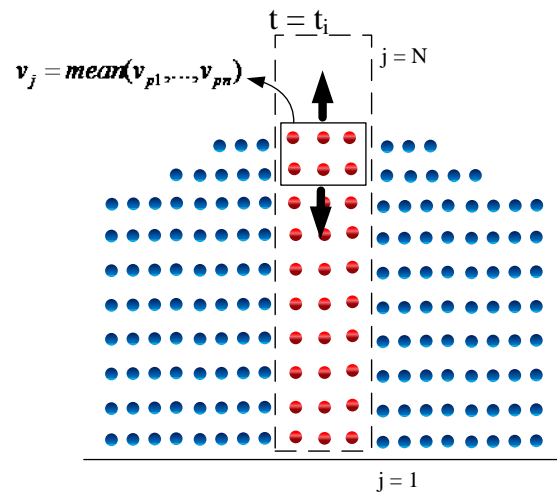

Figure 7 - Schematic of velocity profile measurements from SPH simulations

Fig. 8 illustrates the comparison of temporal averaged cross-shore velocity profiles between the WCSPH model and LDA data.

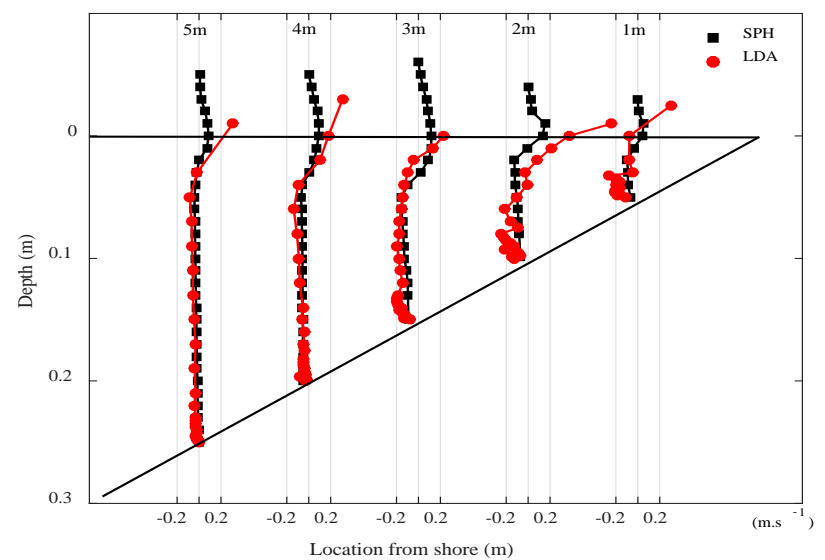

Figure 8 - Comparison between on-offshore temporal-averaged velocity profiles of the SPH model and the LDA measurements for monochromatic waves of $S_{o p}=5 \%$,

The mixing coefficients were calculated for both LDA data and the WCSPH model. The LDA hydrodynamic data along with the depth-varying diffusivities determined from TKE measurements are inputted to the $\mathrm{N}$-zone model. Table 3 compares the shear dispersion coefficients obtained from LDA with the tracer measurements. For the monochromatic waves of 5\% steepness, the $N$-zone dispersion model underestimated the dispersion at the breaker region and in the inner surfzone. The results of the $N$-zone model show that the dispersive mechanism only varies by $1.079 \mathrm{E}^{-3} \mathrm{~m}^{2} / \mathrm{s}$ across the

surfzone $(y=1,2,3 \mathrm{~m})$. 
Table 3 - Comparison of the shear dispersion coefficient determined from LDA data with N-zone model (undertow values and depth-varying diffusivities) with the dye measurements

\begin{tabular}{lcccccccc}
\hline \multirow{2}{*}{ Condition } & \multicolumn{7}{c}{ Shear Dispersion $\left(\mathrm{m}^{2} \cdot \mathrm{s}^{-1}\right)$} \\
\cline { 3 - 9 } & & \multicolumn{7}{c}{ Location from SWL $(\mathrm{m})$} \\
\hline $\mathrm{H}(\mathrm{m})$ & $\mathrm{T}(\mathrm{sec})$ & 1 & 2 & 3 & 4 & 5 & 6 \\
\hline \multirow{3}{*}{0.12} & \multirow{2}{*}{1.2} & $8.94 \mathrm{E}-3$ & $1.03 \mathrm{E}-2$ & $1.02 \mathrm{E}-2$ & $5.64 \mathrm{E}-3$ & $2.20 \mathrm{E}-3$ & $1.93 \mathrm{E}-3$ & Model \\
\cline { 3 - 8 } & & N.A & $1.19 \mathrm{E}-2$ & $1.26 \mathrm{E}-2$ & N.A & $6.93 \mathrm{E}-4$ & N.A & Dye \\
\hline
\end{tabular}

The shear dispersion is calculated for the duration of WCSPH simulations. The $N$-zone model is applied for the data from each time-step of WCSPH model. Fig. 9 shows the temporal variation of mixing coefficient determined for the WCSPH data inside the surfzone (Fig. 9a), at the breaker region (Fig. 9b) and seaward of the breaker region (Fig. 9c). The overall mixing coefficient was determined by averaging the dispersion coefficient over the length of simulation. For each location, the figure shows the temporal variation of $D_{y}$ for $25 \mathrm{sec}$ of the WCSPH simulations (top figure). It also shows the evolution of the dispersion coefficients during a wave cycle (bottom figure). It is evident that for all locations shown in Fig. 9, the SPH model needs a few seconds to produce steady predictions for the dispersion. The small and unrealistic fluctuations in the beginning of the SPH simulations are due to the initial conditions and the fact that the movements of the particles is yet not reached to the measurement location. The results for mixing coefficients confirm the existence and contributions of bore, undertow and bore/undertow collision phases within the wave cycle.

Comparison of the temporal variation of the shear dispersion with the numerical simulations shows that the shear dispersion has very small fluctuations during the undertow phase. The interaction of the incoming bore meeting the seaward moving undertow resulted in a short intense turbulent shear layer, as demonstrated by a rapid increase and decrease in the mixing over the wave cycle (Fig. 9a, b \& c).

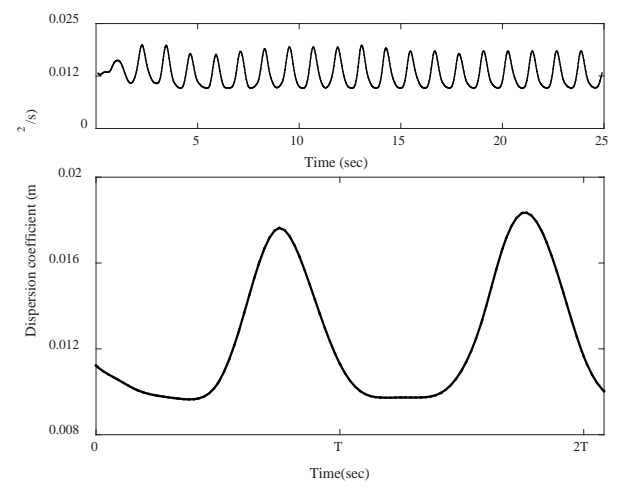

(a)
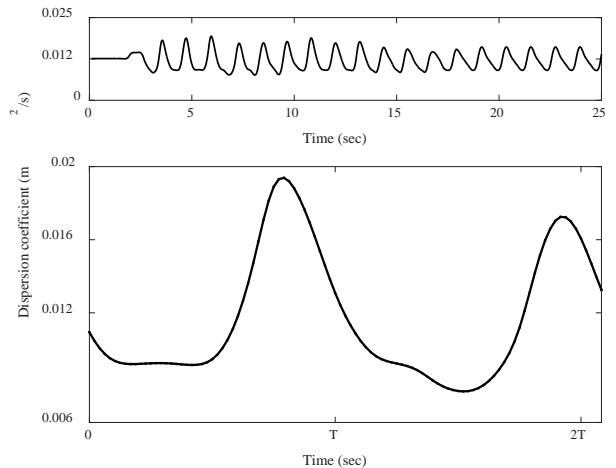

(b)

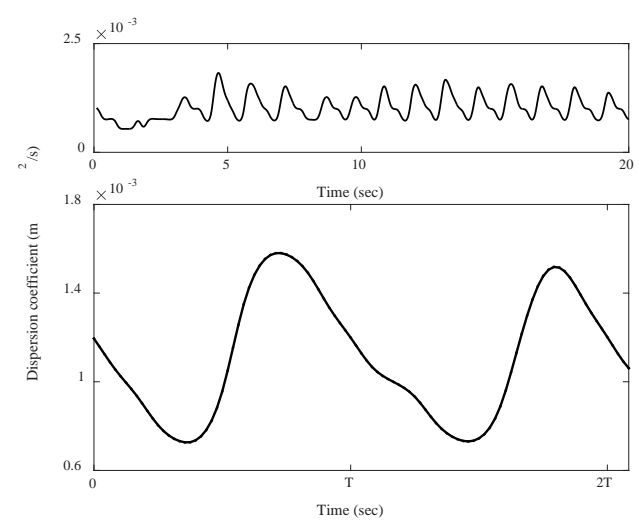

(c)

Figure 9 - Temporal variation of mixing coefficients obtained from WCSPH model for regular waves of $S_{o p}$ $=5 \%$ at a) surfzone $[2 \mathrm{~m}]$, b) breaker region $[3 \mathrm{~m}], \mathrm{c})$ offshore $[5 \mathrm{~m}]$ 
Tables 4 summarize the mixing coefficient determined by using the WCSPH hydrodynamics and the $\mathrm{N}$-zone dispersion model. The overall mixing is also compared to the DHI fluorometric measurements.

Table 4 - Dispersion coefficients from the WCSPH simulations for the monochromatic waves with $H_{o}=$ $0.12 \mathrm{~m}$ and $T=1.2 \mathrm{sec}$

\begin{tabular}{|c|c|c|c|c|c|c|c|}
\hline \multirow{2}{*}{\multicolumn{2}{|c|}{ Condition }} & \multicolumn{6}{|c|}{ Shear Dispersion (m2.s-1) } \\
\hline & & \multicolumn{5}{|c|}{ Location from SWL (m) } & \\
\hline $\mathrm{H}(\mathrm{m})$ & $\mathrm{T}(\mathrm{sec})$ & 1 & 2 & 3 & 4 & 5 & \\
\hline \multirow{2}{*}{0.12} & \multirow{2}{*}{1.2} & 1.12E-02 & 1.20E-02 & 1.22E-02 & 7.10E-03 & $9.98 \mathrm{E}-04$ & WCSPH \\
\hline & & N.A & $1.19 \mathrm{E}-2$ & 1.26E-2 & N.A & $6.93 E-4$ & Dye \\
\hline
\end{tabular}

The mixing coefficients obtained from the temporal variation of shear dispersion from the WCSPH simulations (Tables 4) show that good agreement exists between the numerical model predictions and the mixing coefficients determined for fluorometric tracer measurements of the DHI study. Fig. 10 compares the diffusive and dispersive mechanisms postulated in this paper, obtained from the hydrodynamics measurements and WCSPH simulations with the fluorometric study.
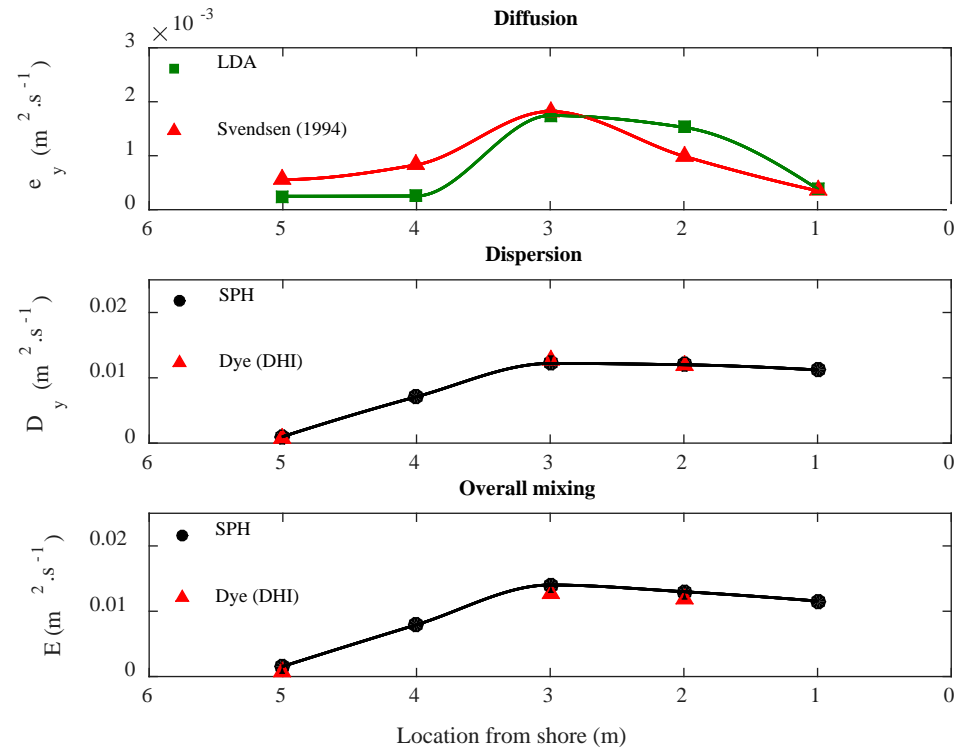

Figure 10 - The diffusive and dispersive mixing mechanisms for the monochromatic waves of $5 \%$ steepness

\section{CONCLUSIONS}

This paper presents investigations focused on understanding and quantifying the nearshore mixing processes due to the effects of waves and currents. The developed theoretical model described the overall mixing coefficient as the sum of the diffusive and dispersive mechanisms due to the wave motion in the nearshore region. The diffusive mechanisms were quantified through measurements as a function of turbulent kinetic energy and the turbulent length-scale. A mathematical approach was adopted to quantify the dispersion coefficient.

Mixing under the combined effects of waves and currents were studied through measurement of hydrodynamic and fluorometric tracing experiments for the monochromatic waves with 5\% offshore steepness. The diffusion and dispersion coefficients for the hydrodynamic data were determined and the results were compared to the mixing coefficients obtained from the tracer measurements. The numerical capabilities of a Lagrangian, particle-based, Smoothed Particle Hydrodynamics method were employed to simulate the wave hydrodynamics in the nearshore region. The numerical results for the wave hydrodynamics were employed to study the shear dispersion in the nearshore region. Very good 
agreement was observed between the mixing coefficient determined from SPH data and the fluorometric tracer study (Fig.10). The results confirmed that dispersive shear mechanism plays significant role in the overall mixing in the nearshore. However, turbulence is still an essential parameter which influences the vertical velocity profile and therefore the dispersion.

\section{REFERENCES}

Abolfathi, S. 2017. Nearshore Mixing due to the effects of waves and current. PhD thesis, University of Warwick.

Abolfathi, S. and J. M. Pearson. 2014. Solute dispersion in the nearshore due to oblique waves, Proceedings of $14^{\text {th }}$ International Conference on Coastal Engineering, ASCE, 1(34), waves 49.

Bowen, A. J. \& D. L. Inman, 1974. Nearshore mixing due to waves and wave-induced currents. Rapp. P.-v. Reun. Cons. int. Explor. Mer, 167: 6-12.

Chikwendu S. C. 1986. Calculation of longshore shear dispersivity using an N-zone model, J. Fluid Mech., 167, 19-30.

Clark D. B., L. Lenain, F. Feddersen, E. Boss, and R. T. Guza. 2014. Aerial imaging of fluorescent dye in the nearshore, J. Atmos. Oceanic Tech., 31, 1410-1421.

Feddersen, F. 2007. Breaking wave induced cross-shore tracer dispersion in the surfzone: Model results and scalings, J. Geophys. Res.,112, C0912.

George, R., R. E. Flick, R. T. GUZA. 1994. Observations of turbulence in the surf zone. J. Geophys. Res., 99, 801-810.

Goring D. G., V. I. Nikora, 2002. Despiking acoustic Doppler velocimeter data. J. of Hydraulic Eng., vol. 128, $117-126$.

Gotoh, H., Shibahara, T. \& T. Sakai, 2001. Sub-particle-scale turbulence model for the MPS method Lagrangian flow model for hydraulic engineering, J. Comp. Fluid Dyn. 9(4), 339- 347.

Grant S. B., J. H. Kim, B. H. Jones, S. A. Jenkins, J. Wasyl, C. Cudaback. 2005. Surf zone entrainment, along-shore transport, and human health implications of pollution from tidal outlets. Journal of Geophysical Research., 110 (C10).

Harris, T. F. W., Jordan, J. M., W. R. McMurry, C. J. Verwey, F. P. Anderson, 1963. Mixing in the surf zone. Int. J. Air Wat. Pollut., 7: 649-67.

Inman D. L., F. J. Tait \& C. E. Nordstrom. 1971. Mixing in the surf zone. J. geophys. Res., 76: 3493.

List E. J., G. Gartrell, C. D. Winant, 1990. 'Diffusion and Dispersion in Coastal Waters', Journal of Hydraulic Engineering, 116(10), 1158-1179

Madsen, O. S. 1971. On the generation of long waves, Journal of Geophysical Research, 76(36).

Monaghan, J.J. 1994. Simulating free surface flows with SPH. Comp. Physics. 110, 399 - 406.

Nadaoka, K., T. Kondoh, 1982. Laboratory measurements of velocity field structure in the surf zone by LDV. Coastal Engng. Japan, 25,125-145.

Pearson, J. M., I. Guymer, L. E. Coastes, J. R. West. 2002. Effect of wave height on on-off shore solute mixing. J. Wtrwy., Port, Coast., and Oc. Engng., ASCE. 1-11.

Pearson, J. M., I. Guymer, L. E. Coastes, J. R. West. 2009. On-off shore Solute Mixing in the SurfZone. J. Wtrwy., Port, Coast., and Oc. Engng., ASCE. Vol 135, No. 4.

Pearson, J. M., I. Guymer, T. V. Karambas., O. S. Petersen. 2006. Laboratory investigation of Mixing in the Nearshore. Proc. 30th Int. Conf. Coastal Eng. San Diego

Spydell M., F. Feddersen, R. T. Guza, W. Schmidt., 2007. Observing surfzone dispersion with drifters, J. Phys. Oceangraphy., 37, 2920-2939.

Svendsen, I. A. \& U. Putrevu. 1994. Near-shore mixing and dispersion. Proc. R. Soc. London, Ser. A, 445, 561-576.

Takewaka, S., S. Misaki, T. Nakamura, 2003. Dye diffusion experiment in a longshore, current field, Coastal Engineering Journal, World Scientific Publishing Company and Japan Society of Civil Engineers. 45(3), 471-487.

Taylor G. I., 1954. The dispersion of matter in a turbulent flow through a pipe. Proc. R. Soc. London, Ser. A, 223, 446-468.

Tseng R. S., 2001. 'On the Dispersion and Diffusion near Estuaries and around Islands', Estuarine Coastal and Shelf Science, v54, 89-100.

Trowbridge J., S. Elgars., 2001. Turbulence measurements in the surfzone, J. Phys.Oceanogr.,31.2403.

Yeganeh-Bakhtiary, A., H. Houshangi, F. Hajivalie, and S. Abolfathi. 2017. A Numerical Study on the Hydrodynamics of Standing Waves in front of Caisson Breakwaters by a WCSPH Model. Coastal Engineering Journal, Volume 59(2): 1750005. 